\title{
Dispersión espacial de Plicopurpura patula pansa en playas rocosas del estado de Guerrero, México
}

\section{Spatial pattern of Plicopurpura patula pansa, in rocky shores of Guerrero, México}

\author{
Sergio García-Ibáñez ${ }^{*}$, Pedro Flores-Rodríguez² ${ }^{2}$ Rafael Flores-Garza² y Arcadio Valdés-González² \\ ${ }^{1}$ Laboratorio de Acuacultura, Unidad Académica de Ecología Marina. Universidad Autónoma de Guerrero. Gran Vía Tropical 20, . 39390 Acapulco, \\ Guerrero, México. \\ ${ }^{2}$ Laboratorio de Acuacultura, Facultad de Ciencias Biológicas. Universidad Autónoma de Nuevo León. Cd. Universitaria, 66450 San Nicolás de los \\ Garza, Nuevo León, México. \\ *Correspondencia: sergariba@yahoo.com.mx
}

Resumen. Plicopurpura patula pansa es un caracol carnívoro característico de las costas rocosas del Pacífico mexicano. Presenta una glándula hipobraquial que secreta un fluido que adquiere una coloración púrpura en presencia de oxígeno y luz solar. Se determinó el arreglo espacial del caracol en playas que difieren en cuanto al tipo de sustrato, exposición al oleaje y pendiente de playa. Se calculó entre 0.1522 y 0.5435 . Las menores fluctuaciones del $\mathrm{I}_{\mathrm{p}}$ se presentaron en 5 playas que tienen en común sustratos fijos, gran cantidad de grietas y oquedades. Las mayores fluctuaciones correspondieron a 4 playas con sustratos móviles, como los cantos rodados.

Palabras clave: costa rocosa, Guerrero, patrón espacial, caracol púrpura.

\begin{abstract}
Plicopurpura patula pansa is a carnivore snail common of the Mexican Pacific rocky shores. Its hypobranchial gland produces a secretion that, in the presence of oxygen and sunlight, becomes purple. We determined the spatial distribution of the snail on beaches that differ in substratum, wave exposure, and the degree substratum's inclination. The average snail density was calculated. Using the Morisita index $\left(\mathrm{I}_{\mathrm{d}}\right)$ and the Morisita standardized index $\left(\mathrm{I}_{\mathrm{p}}\right)$, we found that in $96.3 \%$ of the samples taken, the snails were distributed according to a patchy distribution. The rest of the time, their distribution was random. The $I_{p}$ index oscillated between 0.1522 and 0.5435 . Smaller fluctuations of the $I_{p}$ index were observed in 5 beaches with substrata that were more stable and had a larger number of cracks and crevices than those on beaches with mobile substrata, such as rolled rocks.
\end{abstract}

Key words: rocky shores, Guerrero, spatial pattern, purple snail.

\section{Introducción}

El caracol Plicopurpura patula pansa es un carnívoro característico de las costas rocosas del Pacífico mexicano. En Acapulco Guerrero es una especie dominante de la comunidad (Flores-Rodríguez et al., 2003). Se distribuye desde Baja California Sur hasta Colombia y las islas Galápagos (Keen, 1971) y habita preferentemente en la zona denominada mesolitoral superior (Villalpando, 1986; Villarroel et al., 2000). Este molusco presenta una glándula que secreta un fluido que, en presencia de la luz y el oxígeno, forma un tinte utilizado para colorear tejidos y

Recibido: 13 noviembre 2004; aceptado: 28 marzo 2007 recibe el nombre de "púrpura de Tiro" (Naegel y Cooksey, 2002). En México, desde hace cientos de años, grupos étnicos como los mixtecos y los nahuas han utilizado este tinte para teñir hilos e indumentaria usada por personas de importancia social y/o religiosa.

Trabajos como los de Hernández y Acevedo (1987), Álvarez (1989), Castillo-Rodríguez y Amezcua-Linares (1992), Holguín (1993), Acevedo et al. (1996), Enciso et al. (1998) y Michel-Morfin et al. (2000) registraron un comportamiento gregario del caracol púrpura en campo y laboratorio. León (1989) estudió el patrón espacial de la especie en una playa de Jalisco, México, y determinó que los individuos de la población se distribuían al azar durante gran parte del año y sólo en ciertas fechas se agregaban. 
Los animales no suelen estar distribuidos de manera uniforme; tienden a agregarse debido al comportamiento o a la heterogeneidad del terreno (Andreawartha, 1973). El tipo de dispersión es el resultado de factores intrínsecos y extrínsecos y según Badii et al. (2000) puede ser el resultado de una adaptación evolutiva de los organismos para optimizar recursos, espacio e intercambio genético. En gasterópodos intermareales, la agregación es importante como mecanismo de protección a las altas temperaturas y de reducción de la desecación (Esqueda et al. 2000). El patrón espacial también determina los métodos de muestreo (Krebs, 1999), ya que un mismo método dará resultados muy distintos según la disposición de la población (Rabinovich, 1980).

El propósito del presente estudio fue determinar el patrón espacial de $P$. patula pansa en playas rocosas del estado de Guerrero que difieren en la exposición al oleaje, en el tipo de sustrato y en la pendiente de playa.

\section{Materiales y métodos}

Área de estudio. El estado de Guerrero se ubica en la porción sureste de México. El trabajo se desarrolló en 9 playas rocosas. La descripción de las playas se realizó de acuerdo con las cartas geológicas del Instituto Nacional de Estadística, Geografía e Informática, con Mottana et al. (1980) y con observaciones realizadas durante la investigación.

Punta Maldonado (PM). Se ubica entre los $16^{\circ} 19^{\prime}$ $26.7^{\prime \prime} \mathrm{N}$ y $98^{\circ} 34^{\prime} 4.6^{\prime \prime} \mathrm{O}$. Es una playa con oleaje intenso o expuesta. El sustrato es de textura semiáspera al tacto y presenta fracturas y gran cantidad de oquedades de diámetro y profundidad variables. La pendiente promedio registrada es de $30.09^{\circ}$.

Las Peñitas (LP). Su ubicación es entre los $16^{\circ}$ $33^{\prime} 14.4^{\prime \prime} \mathrm{N}$ y $98^{\circ} 46^{\prime} 22.0^{\prime \prime} \mathrm{O}$. Es una playa con oleaje suave o protegida. Macizo rocoso de textura áspera al tacto, con gran cantidad de protuberancias, grietas y oquedades. La pendiente promedio es de $51.62^{\circ}$.

Playa Ventura (PV). Se ubica entre los $16^{\circ} 32^{\prime} 8.3^{\prime \prime} \mathrm{N}$ y $98^{\circ} 54^{\prime} 44.6^{\prime \prime}$ O. Es una playa expuesta. Macizo rocoso de textura áspera al tacto, con gran cantidad de grietas y oquedades. La pendiente promedio registrada es de $59.6^{\circ}$.

Zoológico (ZO). Se ubica en la isla La Roqueta, Acapulco, entre los $16^{\circ} 42^{\prime} 11.2^{\prime \prime} \mathrm{N}$ y $99^{\circ} 54^{\prime} 8.8^{\prime \prime} \mathrm{O}$. Playa expuesta. Macizo rocoso fijo, con textura áspera al tacto y gran cantidad de grietas y oquedades. La pendiente promedio registrada es de $35.18^{\circ}$.

Palmitas (PA). Se ubica en la isla La Roqueta, Acapulco, entre los $16^{\circ} 49^{\prime} 25.6^{\prime \prime} \mathrm{N}$ y $99^{\circ} 54^{\prime} 41.2^{\prime \prime} \mathrm{O}$. Playa protegida. Macizo rocoso de textura áspera al tacto y gran cantidad de grietas y oquedades. La pendiente promedio es de $36.4^{\circ}$.

Tlacoyunque (TL). Ubicada entre los $17^{\circ} 15^{\prime} 14.6^{\prime \prime} \mathrm{N}$ y $101^{\circ} 00^{\prime} 46^{\prime \prime} \mathrm{O}$. Playa expuesta. Macizo rocoso fijo de textura áspera al tacto, con zonas donde hay gran cantidad de grietas y oquedades así como zonas de complejidad mínima. La pendiente promedio es de $49.6^{\circ}$.

Barra de Potosí expuesta (BP1). Se ubica en la Punta el Potosí entre $\operatorname{los} 17^{\circ} 32^{\prime} 12.5^{\prime \prime} \mathrm{N}$ y $101^{\circ} 26^{\prime} 54.6^{\prime \prime} \mathrm{O}$. Playa expuesta. Presenta una combinación de macizos rocosos fijos de textura semiáspera y cantos rodados con superficies lisas. La pendiente promedio es de $22.7^{\circ}$.

Barra de Potosí protegida (BP2). Se ubica en Punta el Potosí entre los $17^{\circ} 32^{\prime} 17.4^{\prime \prime} \mathrm{N}$ y $101^{\circ} 27^{\prime} 19.1^{\prime \prime} \mathrm{O}$. Playa protegida. Se compone exclusivamente de cantos rodados de superficie lisa, con pequeñas oquedades y casi nula presencia de grietas y fisuras. La pendiente promedio es de $15.3^{\circ}$.

Troncones (TR). Se ubica entre los $17^{\circ} 47^{\prime} 35.0^{\prime \prime} \mathrm{N} \mathrm{y}$ $101^{\circ} 44^{\prime} 46.6^{\prime \prime} \mathrm{O}$. Playa protegida. Presenta macizos fijos de textura áspera y gran cantidad de fisuras y grietas. La pendiente promedio es de $28.4^{\circ}$.

Trabajo de campo. El muestreo se realizó durante la marea baja y a la luz del día en septiembre y diciembre 2000, marzo, junio, septiembre y diciembre 2001. Para delimitar el área se empleó una línea de $30 \mathrm{~m}$ de largo paralela a la costa con $2 \mathrm{~m}$ de ancho. De acuerdo con el criterio de Stephenson y Stephenson (1949), la zona en la que se llevó a cabo el muestreo fue la franja mesolitoral. Verticalmente se precisaron 2 niveles: nivel I, aquel cuyo límite inferior colindó con la zona de balanos; nivel II, aquel cuyo límite superior fue la zona de neritas y litorinas. Con base en el ajuste de datos de campo previamente obtenidos, a distribuciones de probabilidad Poisson y binomial negativa, se determinó un tamaño de muestra de $20 \mathrm{~m}^{2}$, con nivel de confianza $\alpha=0,10$ y error estándar de $30 \%$. El muestreo fue sistemático (Cochran, 1980), con unidad de muestra de $1 \mathrm{~m}^{2}$.

La rutina consistió en colocar el cuadrante en un punto seleccionado en el nivel I y contabilizar todos los ejemplares de $P$. p. pansa. Posteriormente se colocó en el nivel II, realizando la misma operación. Al concluir esas 2 unidades de muestra se dejó un espacio de $2 \mathrm{~m}$, instalando nuevamente el cuadrante en el nivel I y en el II, respectivamente; la operación se repitió de manera sucesiva hasta obtener un total de $20 \mathrm{~m}^{2}$.

Análisis de datos. Se calculó la densidad promedio de organismos durante todo el estudio, dividiendo el número de caracoles registrados en cada playa, por el total de metros cuadrados contabilizados.

Con las frecuencias de caracoles se determinó el patrón espacial del caracol de tinte en cada playa rocosa, 
y la fecha de muestreo mediante los siguientes índices de dispersión: a) varianza/media $(\mathrm{V} / \mathrm{m})$; b) Morisita $\left(I_{d}\right)$, y $\left.c\right)$ estandarizado de Morisita ( $\left.I_{p}\right)$ (Krebs, 1999).

Los índices $V / m$ e $I_{d}$, toman valor de 1 para poblaciones distribuidas de manera aleatoria; valores menores a 1, para poblaciones con tendencia a la uniformidad, y valores mayores a 1 para poblaciones agregadas. En el caso del $I_{d}$, la evaluación de la significancia estadística se realizó con la prueba ji-cuadrada $\left(x^{2}\right)$, con $n-1$ grados de libertad y probabilidad de error $\alpha=0.05$.

El $\left(I_{p}\right)$ fluctúa de -1.0 a 1.0 con límites de confianza al $95 \%$, si $I_{p} \geq 0.5$. Patrones aleatorios dan un valor de $I_{p}$ igual a cero; patrones agregados por arriba de cero; patrones uniformes por debajo de cero.

\section{Resultados}

La densidad promedio obtenida para cada playa rocosa durante el estudio, fluctuó de 0.89 a 10.26 caracoles $/ \mathrm{m}^{2}$ (Fig. 1). El valor mínimo que registró el índice $(\mathrm{V} / \mathrm{m})$, fue 1.22 , el cual se determinó para la población de caracoles de la playa Punta Maldonado en septiembre 2001; el valor máximo fue 14.86, que se registró en Zoológico en la fecha de muestreo citada anteriormente (Cuadro 1).

Al igual que el índice anterior, todos los valores del $\left(I_{d}\right)$ fueron mayores a la unidad y se determinó que de 54 pruebas, 47 resultaron estadísticamente significativas (Ji-cuadrada, $p \leq 0.05$ ). El valor mínimo que se registró fue 1.05 y el máximo 2.05; ambos se determinaron en Punta Maldonado durante las fechas de septiembre 2001 y 2000, respectivamente. Punta Maldonado, Piedra de Tlacoyunque, Barra de Potosí expuesta y Barra de Potosí protegida fueron las playas donde al menos en una fecha se registraron valores que no difirieron significativamente de la unidad (Cuadro 2).

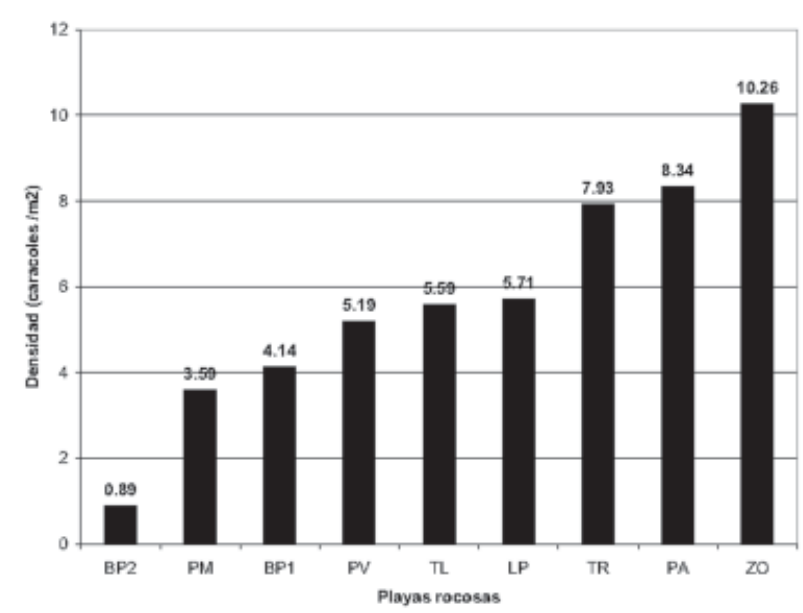

Figura 1. Densidad promedio del caracol Plicopurpura patula pansa, registrada durante seis fechas de muestreo en nueve playas rocosas.

Los valores del $\left(I_{p}\right)$, fluctuaron entre 0.1522 (Punta Maldonado en septiembre 2001) y 0.5435 (Barra de Potosí protegida en diciembre 2000). Las menores fluctuaciones del $I_{p}$, se apreciaron entre los valores que se registraron en las playas Las Peñitas, Playa Ventura, Zoológico, Palmitas y Troncones. Aunque algunas se encuentran separadas por cientos de kilómetros, todas estas playas presentan sustratos fijos con gran cantidad de grietas y oquedades así como pendientes promedio entre 28.4 y $59.6^{\circ}$. En cuanto a la intensidad del oleaje, 3 de dichas playas son protegidas y 2 son expuestas.

Se observaron fluctuaciones más amplias del $I_{p}$ en los valores correspondientes a las poblaciones de caracoles de las playas Barra de Potosí protegida, Piedra de Tlacoyunque y Punta Maldonado, donde se percibieron caídas abruptas del índice en los meses de marzo, junio

Cuadro 1. Valores de la razón V/m para el caracol Plicopurpura patula pansa, en 9 playas rocosas del Estado de Guerrero, México, durante 6 fechas de muestreo

\begin{tabular}{lcccccc}
\hline \multicolumn{1}{c}{ Fecha } & Sep-00 & Dic-00 & Mar-01 & Jun-01 & Sep-01 & Dic-01 \\
\hline Punta Maldonado & 2.88 & 3.03 & 1.94 & 2.25 & 1.22 & 1.26 \\
Las Peñitas & 5.22 & 6.11 & 5.36 & 3.56 & 6.22 & 4.82 \\
Playa Ventura & 2.74 & 4.58 & 3.10 & 2.93 & 2.32 & 5.69 \\
Zoológico & 5.43 & 4.06 & 2.21 & 4.18 & 14.86 & 2.37 \\
Palmitas & 2.004 & 2.02 & 2.45 & 4.50 & 3.98 & 2.58 \\
Piedra de Tlacoyunque & 1.57 & 3.80 & 1.51 & 1.28 & 3.04 & 7.16 \\
Barra de Potosí Exp. & 1.49 & 1.64 & 2.22 & 2.99 & 2.28 & 3.55 \\
Barra de Potosí Prot. & 1.89 & 2.18 & 1.39 & 1.52 & 2.05 & 1.68 \\
Troncones & 1.88 & 2.57 & 2.44 & 10.76 & 7.04 & 1.87 \\
\hline
\end{tabular}


Cuadro 2. Valores del índice de Morisita $I_{d}$, para el caracol Plicopurpura patula pansa, en 9 playas rocosas del estado de Guerrero, México, durante 6 fechas de muestreo

\begin{tabular}{|c|c|c|c|c|c|c|}
\hline Fecha & Sep-00 & Dic-00 & Mar-01 & Jun-01 & Sep-01 & Dic-01 \\
\hline Punta Maldonado & $2.05 *$ & $1.38 *$ & $1.24 *$ & $1.53 *$ & 1.05 & 1.06 \\
\hline Las Peñitas & $1.72 *$ & $1.68 *$ & $1.73 *$ & $1.52 *$ & $1.79 *$ & $1.78 *$ \\
\hline Playa Ventura & $1.44 *$ & $1.85^{*}$ & $1.47 *$ & $1.34 *$ & $1.26 *$ & $1.53 *$ \\
\hline Zoológico & $1.45^{*}$ & $1.29 *$ & $1.12 *$ & $1.30 *$ & $2.03 *$ & $1.13 *$ \\
\hline Palmitas & $1.09 *$ & $1.11 *$ & $1.21 *$ & $1.45^{*}$ & $1.43 *$ & $1.16^{*}$ \\
\hline Piedra de Tlacoyunque & $1.12 *$ & $1.56^{*}$ & 1.13 & 1.10 & $1.32 *$ & $1.51 *$ \\
\hline Barra de Potosí Exp. & 1.09 & $1.13 *$ & $1.28 *$ & $1.37 *$ & $1.71 *$ & $1.61 *$ \\
\hline Barra de Potosí Prot. & $3.81 *$ & $4.76 *$ & 1.47 & 1.47 & $2.0^{*}$ & $1.40 *$ \\
\hline Troncones & $1.09 *$ & $1.23 *$ & $1.30 *$ & $1.89 *$ & $1.57 *$ & $1.13 *$ \\
\hline
\end{tabular}

* Significativos al rechazar $\mathrm{H}_{\mathrm{o}} \operatorname{con} \alpha=0.05$

Cuadro 3. Valores del índice estandarizado de Morisita $I$, para el caracol Plicopurpura patula pansa, en 9 playas rocosas del estado de Guerrero, México, durante 6 fechas de muestreo

\begin{tabular}{lcccccc}
\hline \multicolumn{1}{c}{ Fecha } & Sep-00 & Dic-00 & Mar-01 & Jun-01 & Sep-01 & Dic-01 \\
\hline Punta Maldonado & 0.5173 & 0.5065 & 0.5014 & 0.5059 & 0.1522 & 0.1752 \\
Las Peñitas & 0.5157 & 0.5154 & 0.5161 & 0.5099 & 0.5181 & 0.5167 \\
Playa Ventura & 0.5067 & 0.5180 & 0.5081 & 0.5056 & 0.5031 & 0.5117 \\
Zoológico & 0.5099 & 0.5058 & 0.5013 & 0.5062 & 0.5257 & 0.5016 \\
Palmitas & 0.5006 & 0.5008 & 0.5028 & 0.5095 & 0.5085 & 0.5023 \\
Piedra de Tlacoyunque & 0.3913 & 0.5109 & 0.3502 & 0.1934 & 0.5055 & 0.5118 \\
Barra de Potosí Exp. & 0.3413 & 0.4362 & 0.5030 & 0.5063 & 0.5083 & 0.5116 \\
Barra de Potosí Prot. & 0.5150 & 0.5435 & 0.2718 & 0.3577 & 0.5084 & 0.4638 \\
Troncones & 0.5004 & 0.5033 & 0.5039 & 0.5218 & 0.5133 & 0.5006 \\
\hline
\end{tabular}

y septiembre de 2001, respectivamente; a partir de esas fechas, se observó tendencia ascendente en las 3 playas. Por lo que respecta a Barra de Potosí expuesta, los valores del $I_{p}$ siempre presentaron tendencia ascendente, que se mantuvo constante a partir de marzo de 2001. Las 4 playas son diferentes entre sí, debido a la variedad de sustratos así como a la pendiente de playa. Punta Maldonado y Barra de Potosí protegida presentan sustratos móviles con diferente estructura y complejidad; en la primera se encuentran gran cantidad de oquedades distribuidas de manera uniforme por toda el área, mientras que en la segunda, el sustrato está compuesto de superficies lisas y son escasas las oquedades. Piedra de Tlacoyunque tiene sustratos fijos con gran cantidad de grietas, así como rocas con superficie porosa pero sin fisuras, grietas u oquedades. En Barra de Potosí expuesta, hay una combinación de grandes bloques de piedra fijos con grietas, y cantos rodados lisos con pocas oquedades. Por lo que respecta a la intensidad del oleaje,
3 de estas playas son expuestas y sólo una es protegida. La pendiente promedio en estas playas fluctúa de $15.3^{\circ} \mathrm{a}$ $49.6^{\circ}$ (Cuadro 3 ).

\section{Discusión}

Durante la recolecta, los caracoles con frecuencia se encontraron agrupados en el interior de grietas y oquedades, mostrando actividades de reproducción y alimentación o protegiéndose de la insolación solar directa, siendo poco frecuente observar durante el día individuos aislados sobre la roca desnuda y expuesta.

Tanto la razón $V / m$ como el $I_{d}$ siempre fueron mayores a la unidad, por lo que de acuerdo a Krebs (1999), existe evidencia de agregación del caracol de tinte. En todos los casos, el índice $I_{p}$ presentó valores por arriba de cero, manifestando un comportamiento gregario de la especie, 
lo que coincidió con los resultados de $V / m$ e $I_{d}$. En particular, el $I_{p}$ es altamente recomendado en muestreos por cuadrantes porque no lo afectan ni la densidad poblacional ni el tamaño de muestra (Krebs, 1999).

Por lo anterior, es evidente que en las playas de estudio, el caracol de tinte Plicopurpura patula pansa presentó un patrón espacial agregado, con excepción de una sola playa con 2 fechas en las que el patrón espacial se definió como aleatorio. En ningún caso hubo tendencia de las poblaciones del caracol de tinte hacia un patrón espacial uniforme.

Andreawartha (1973) manifestó que los animales no se dispersan de modo uniforme y tienden a formar agregaciones. Los resultados de esta investigación muestran que el caracol de tinte mantiene un patrón espacial agregado a través del tiempo, aún cuando las características del sustrato y exposición de cada playa rocosa varían entre si.

Las menores fluctuaciones de valores del $I_{p}$, se observaron en 5 playas que tuvieron en común sustratos fijos, superficies porosas o ásperas con gran cantidad de grietas y oquedades. Las mayores fluctuaciones del $I_{p}$, fueron para 4 playas con características del sustrato muy diferentes entre sí, donde resaltan sustratos fijos y/o móviles, superficies lisas o semiásperas así como una complejidad variable que va desde muy pocas oquedades hasta gran cantidad de grietas. De esta manera, las agregaciones del caracol de tinte se mantienen más firmes en sitios donde el sustrato les proporciona una mayor protección y mejor adhesión a la superficie. Por otro lado, en lugares donde la movilidad del sustrato podría provocarles daño (en zonas de cantos rodados se llegaron a detectar caracoles con conchas rotas) y en aquellos donde la superficie es lisa, las variaciones de la agregación resultan evidentes.

Por lo que respecta a la exposición al oleaje, se observó que en las playas donde la fluctuación de los valores del $I_{p}$ fue menor,3 fueron protegidas y 2 expuestas. Por el contrario, donde la fluctuación del mismo índice fue mayor, 3 playas fueron expuestas y una protegida. De esta manera, la intensidad del oleaje podría indicar cierto efecto, ya que las playas donde las poblaciones de caracol presentaron mínimas variaciones del $I_{p}$ estuvieron más relacionadas con oleaje suave, mientras que los sitios donde se presentaron mayores fluctuaciones del mismo índice estuvieron más relacionados con oleaje intenso. Sin embargo, aún con las tendencias anteriores, no es posible demostrar de manera contundente el efecto de la exposición al oleaje sobre la dispersión espacial de la especie.

En cuanto a la pendiente de playa, en el grupo de 5 sitios donde se registró menor fluctuación del $I_{p}, 4$ presentaron pendientes promedio de 35.18 a $59.6^{\circ}$, y el restante $28.4^{\circ}$. En el grupo de 4 sitios con mayor fluctuación del mismo índice, 3 presentaron pendientes entre 15.3 y $30.09^{\circ}$, y el restante $49.6^{\circ}$. Lo anterior denota una tendencia a que las menores fluctuaciones del $I_{p}$ se presenten en sitios con pendiente promedio mayor a $35^{\circ}$. Sin embargo, dicho efecto parece estar en estrecha asociación con la estructura y complejidad del sustrato, ya que en la playa Troncones se registró una inclinación de $28.4^{\circ}$ y en todos los casos, el patrón espacial de la especie fue agregado, mientras que en Piedra de Tlacoyunque la inclinación o pendiente registrada fue de $49.6^{\circ}$ y al menos en una fecha se llegó a presentar una tendencia de dispersión al azar de la especie. Jones y Reynolds (en Sutherland, 1996), comentan que la pendiente tiene un efecto drástico sobre plantas y animales y causa un efecto notable en la zona intermareal.

Otros estudios han descrito un comportamiento gregario de $P$. p. pansa relacionado con ciertas características del hábitat, ya que registraron organismos en lugares que sirven de refugio, protección y zonas de ovipostura, como grietas, oquedades y fisuras (CastilloRodríguez y Amezcua-Linares, 1992; Enciso et al., 1998; Acevedo et al., 1996). El gregarismo por alimentación ha sido comentado por Álvarez (1989) y relacionado con la reproducción por Hernández y Acevedo (1987), Holguín (1993) y Michel-Morfín et al. (2000).

En trabajos como los de Spight (1976 y 1978) se señala que las hendiduras son un elemento importante del hábitat; Menge y Lubchenco (1981) remarcan que los espacios tridimensionales (hoyos y oquedades) tienen importancia como refugio, ya que en superficies abiertas los organismos pueden experimentar calor excesivo y desecación, y además el oleaje puede removerlos. En cuanto a la exposición al oleaje, estudios como los de Hernández y Acevedo (1987), Álvarez (1989), CastilloRodríguez (1992) y Reyes (1993), resaltan que P. patula pansa presenta adaptaciones, como poca ornamentación de la concha, ángulo pequeño de la espira y gran abertura pedal, que proveen al caracol de una mejor adhesión al sustrato y le permite habitar zonas expuestas al fuerte oleaje. Aguilar-Cruz (2003) describe que en el epitelio del pie y manto abundan células calciformes, cuyas secreciones ayudan, entre otras múltiples funciones, a una mejor adhesión al sustrato.

Por lo anterior y de acuerdo con los resultados del presente trabajo, el caracol de tinte muestra un patrón espacial agregado en las áreas de estudio, está adaptado para vivir en zonas expuestas, y mientras habite áreas de sustratos fijos, las variaciones del oleaje pueden tener un efecto menor sobre su disposición en el espacio. La relación entre la pendiente de playa, estructura y complejidad del sustrato, pueden mostrar un efecto mayor sobre el grado de agregación que la intensidad del oleaje.

El único estudio que aborda el patrón espacial de 
Plicopurpura patula pansa de manera metodológica es el de León (1989), en una playa ubicada en Bahía de Cuastecomate, Jalisco, México. El autor realizó un muestreo en un área de $10 \mathrm{~m}^{2}$ y trabajó con $\mathrm{V} / \mathrm{m}$ y el $\left(I_{d}\right)$. Determinó que durante la mayor parte de su estudio, la población presentó una distribución al azar y en agosto, septiembre, noviembre y enero, un patrón agregado.

Los resultados de la presente investigación contrastan con los obtenidos por León (1989), debido a que los 3 índices de dispersión espacial utilizados en el presente estudio coincidieron en que sólo los conjuntos de datos de las fechas de septiembre y diciembre de 2001 en la playa Punta Maldonado mostraron una disposición de los caracoles al azar. Esto es, del $100 \%$ de las observaciones realizadas durante 6 fechas de muestreo en 9 playas rocosas de la costa del estado de Guerrero, el 96.3\% (52 de 54 valores por cada índice) indicó un patrón espacial agregado de la especie y el $3.7 \%$ restante, un patrón aleatorio.

Se sugieren 3 posibles explicaciones: $a$ ) diferencias en el método de muestreo; b) características propias del sitio de estudio, y c) diferencias en la densidad de caracoles.

En cuanto al primer inciso, aunque existen puntos en que ambos métodos de muestreo pueden ser coincidentes (diseño del muestreo y aplicación de los índices $\mathrm{V} / \mathrm{m}$ e $\left.I_{d}\right)$, difieren en el tamaño de la unidad de la muestra, en el número de unidades y en la frecuencia de la obtención de información. En la presente investigación, el uso del $\left(I_{p}\right)$, confirmó los resultados obtenidos por $V / m$ e $I_{d}$.

En cuanto al segundo inciso, aunque existe una diferencia latitudinal, en las características de su playa de estudio, León (1989) describe una combinación de sustratos, entre otros, algunos que pueden tener cierta movilidad en función del oleaje. En este sentido, existiría cierta similitud con las playas Barra de Potosí expuesta y Barra de Potosí protegida del presente trabajo, donde se llegaron a registrar valores del $I_{p}$ bajos, como $0.27 \mathrm{y}$ 0.34. Punta Maldonado, también con características muy particulares del sustrato, presentó los 2 valores más bajos del $I_{p}$, y en esas fechas (septiembre y diciembre de 2001), el patrón espacial del organismo se determinó al azar.

En cuanto a la densidad de organismos, León (1989) registró de 0.2 a 2.7 caracoles $/ \mathrm{m}^{2}$. Taylor $(1971 ; 1984)$ menciona que a muy bajas densidades y en conjuntos de muestras en las que se presenta un solo individuo, se pueden detectar dispersiones al azar. En el presente trabajo, en la playa Barra de Potosí protegida, se presentó la densidad promedio más baja $\left(0.89\right.$ caracoles $\left./ \mathrm{m}^{2}\right)$, observándose una disminución del $I_{p}$ en 2 fechas de muestreo, y en las mismas ocasiones el $\left(I_{d}\right)$ no percibió diferencias significativas de la unidad.

Puede afirmarse que en las 9 playas estudiadas, el patrón de dispersión espacial de P. p. pansa es agregado, lo que puede considerarse una característica de la población, ya que se presentó en playas diferentes en lo que respecta tanto a características asociadas al sustrato, como a exposición al oleaje. El comportamiento gregario puede considerarse uno de los factores que contribuyen al éxito que exhibe el caracol de tinte como especie dominante en dichos lugares.

\section{Agradecimientos}

Agradecemos al Consejo Nacional de Ciencia y Tecnología (CONACYT) y a la Fundación TELMEX por el apoyo y becas otorgadas para la realización de la presente investigación. También nuestro agradecimiento para los licenciados en ecología marina Claudia Ruiz Hernández, Norma Lidia Cruz Ramírez, Alfredo Hernández Maldonado, Alfonso Montaño Rivera y Darío Flores Ávalos, por la ayuda prestada en las labores de campo e investigación.

\section{Literatura citada}

Acevedo, G. J., M. A. Escalante y M. Turok. 1996. El caracol púrpura. In Pesquerías relevantes de México, tomo II,. Secretaría del Medio Ambiente, Recursos Naturales y Pesca, México, D.F. p. 1079-1100.

Aguilar-Cruz, C. A. 2003. Histología y función del epitelio del pie y manto del caracol de tinta Plicopurpura pansa (Gould, 1853) (Prosobranchia: Muricoidea). In Resúmenes del IX Congreso de Asociación de Investigadores del Mar de Cortés y III Simposium Internacional sobre el Mar de Cortés, del 5 al 9 de mayo. La Paz, Baja California Sur, p. 2.

Álvarez, D. A. S. 1989. Relaciones ecológicas y algunos aspectos poblacionales del caracol Purpura pansa Gould, 1853 en la Costa del Estado de Michoacán, México. Tesis, Facultad de Biología, Universidad Michoacana de San Nicolás de Hidalgo, Morelia, Michoacán. 124 p.

Andreawartha, H. G. 1973. Introducción al estudio de poblaciones animales. Alhambra, México, D.F. 332 p.

Badii, M. H., A. E. Flores, R. Foroughbakhch y H. Quiróz. 2000. Fundamentos de muestreo. In Fundamentos y perspectivas de control biológico, M. H. Badii,, A. E. Flores y L. J. Galán W. (eds.). Universidad Autónoma de Nuevo León, San Nicolás de los Garza, p. 129-153.

Castillo-Rodríguez, Z. G. 1992. Combinatio Nova de Plicopurpura pansa (Gould, 1853) (Prosobranchia, Muricoidea). Anales del Instituto de Ciencias del Mar 
y Limnología, Universidad Nacional Autónoma de México 19:103-111.

Castillo-Rodríguez, Z. G. y F. Amezcua-Linares. 1992. Biología y aprovechamiento del caracol morado Plicopurpura pansa (Gould, 1853) (Gastropoda: Neogastropoda) en la costa de Oaxaca, México. Anales del Instituto de Ciencias del Mar y Limnología, Universidad Autónoma de México 19:223-234.

Cochran, W.G. 1980. Técnicas de muestreo. CECSA, México, D.F. 513 p.

Enciso, E. C., V. M. Ramírez H., N. A. Tirado y A. Vallarta P. 1998. Evaluación de la población y épocas de reproducción del caracol Plicopurpura pansa (Gould, 1853), en Mazatlán, Sinaloa, México. Tesis, Facultad de Ciencias del Mar Universidad Autónoma de Sinaloa, Mazatlán, 56 p.

Esqueda, M. C., E. Ríos-Jara, J. E. Michel-Morfin y V. Landa-Jaime. 2000. The vertical distribution and abundance of gastropods and bivalves from rocky beaches of Cuastecomate Bay, Jalisco, México. Revista Biología Tropical 48:765-775.

Flores-Rodríguez, P., R. Flores-Garza, S. GarcíaIbáñez, y A. Valdés-González. 2003. Riqueza y diversidad de la malacofauna del mesolitoral rocoso de la Isla La Roqueta, Acapulco, Guerrero, México. Ciencia, Universidad Autónoma de Guerrero, época II, 11:5-14.

Hernández, C. E. y G. J. Acevedo. 1987. Aspectos poblacionales y etnobiológicos del caracol Purpura pansa, Gould, 1853 en la costa de Oaxaca. Tesis Facultad de Ciencias, Universidad Nacional Autónoma de México, México D.F. 139 p.

Holguín, Q. O. E. 1993. Distribución, abundancia y composición peso-talla de Purpura pansa (Mollusca Gastropoda) en Isla Socorro, Archipiélago Revillagigedo, Mexico. Zoología Informa. Escuela Nacional de Ciencias Biológicas, Instituto Politécnico Nacional 25:24-33

Jones, J. C. y J. D. Reynolds. 1996. Environmental variables. In Ecological census techniques. A handbook, W. J. Sutherland (ed.). Cambridge University, London. 336 p.

Keen, A. M. 1971. Sea shells of tropical West America. Stanford University Press, California. 1064 p.

Krebs, C. J. 1999. Ecological methodology. Longman, Menlo Park, California. 620 p.

León, A. H. G. 1989. Estructura poblacional, producción y tiempo de recuperación del tinte de Purpura pansa Gould, 1853 (Gasterópoda: Thaididae) en algunas playas rocosas de la bahía Cuastecomate, San Patricio Melaque, Jalisco, México. Tesis, Facultad de Ciencias, Universidad de Guadalajara, Jalisco, 107 p.

Menge, B. A. y J. Lubchenco. 1981. Community organization in temperate and tropical rocky intertidal habitats: prey refuges in relation to consumer pressure gradients. Ecological Monographs 51:429-450.

Michel-Morfin, J. E., E. A. Chávez y V. Landa. 2000. Population parameters and dye yield of the purple snail Plicopurpura pansa (Gould, 1853) of West Central México. Journal of Shellfish Research 19:919-925.

Mottana, A., R. Crespi y G. Liborio. 1980. Guía de minerales y rocas. Grijalbo, Barcelona.605 p.

Naegel, L. y C. J. Cooksey. 2002. Tyrian purple from marine muricids, specially from Plicopurpura pansa (Gould, 1853). Journal of Shellfish Research, 21:193200.

Rabinovich, J. E. 1980. Introducción a la ecología de poblaciones animales. CECSA, México, D.F. 313 p.

Reyes, A. S. C. 1993. Estimación poblacional, producción, foto-oxidación y rendimiento del tinte del caracol Purpura pansa (Gould, 1853) de la zona sur del litoral rocoso de Jalisco. Tesis, Facultad de Ciencias Biológicas, Universidad de Guadalajara, Jalisco, 115 p.

Spight, T. M. 1976. Censuses of rocky shore prosobranchs from Washington and Costa Rica. The Veliger 18:309-317.

Spight, T. M. 1978. Temporal changes in a tropical rocky shore snail community. The Veliger 21:137-143.

Stephenson, T. A. y A. Stephenson. 1949. The universal features of zonation between tide-marks on rocky coasts. Journal of Ecology 37:289-305.

Taylor, L. R. 1971. Aggregation as a species characteristic. In Statistical ecology, vol. 1., G. P., Pielou and W. E. Waters (eds.). Pennsylvania State University Press, University Park, State College. 582 p.

Taylor, L. R. 1984. Assessing and interpreting the spatial distribution of insect population. Annual Review of Entomology 29:321-358.

Villalpando, C. E. 1986. Diversidad y zonación de moluscos de facie rocosa, Isla Roqueta, Acapulco Guerrero. Tesis Facultad de Ciencias, Universidad Nacional Autónoma de México, México, D.F. 150 p.

Villarroel, M. M., A. Magaña M., B. Gómez C., O. del Río Z., J. Lucio P. y J. Sánchez S. 2000. Diversidad de moluscos en el litoral rocoso de Michoacán, México. Mexicoa 2:54-63. 